\title{
Painful hips in a patient on long-term steroid treatment
}

\author{
J P Y Lay, R W Whitehouse
}

A 60-year-old Asian man on long-term steroid treatment for chronic active hepatitis presented with pain in both hips. Initial pelvic X-ray was considered unremarkable (figure 1A) and a magnetic resonance imaging (MRI) scan was requested (figure 2). A repeat X-ray was also obtained just prior to the MRI scan, 4 weeks after the initial X-ray (figure 1B).
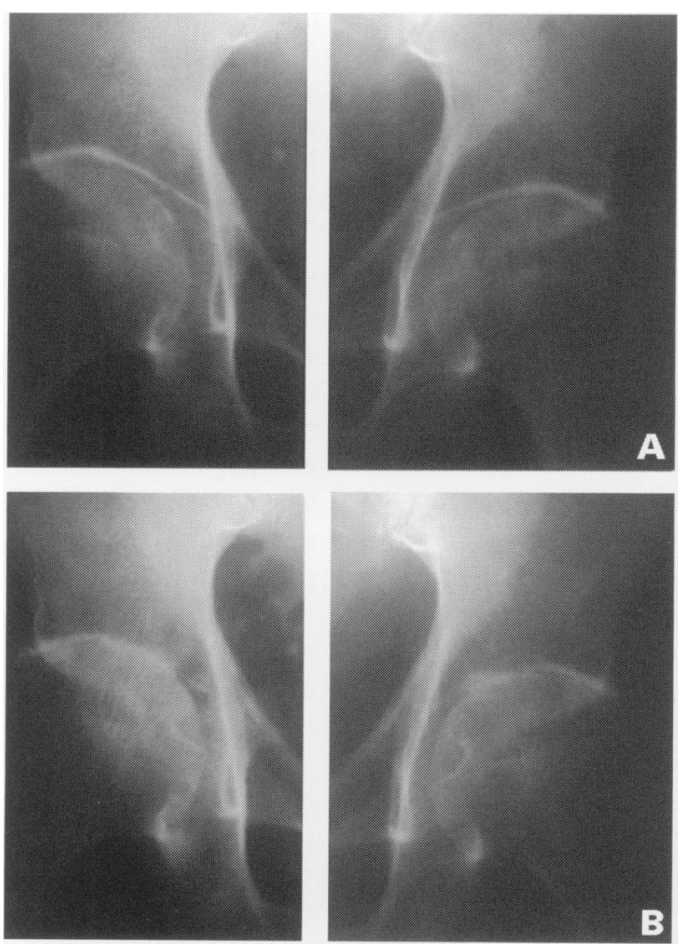

Figure 1 (A) Initial pelvic X-ray; (B) 4 weeks later
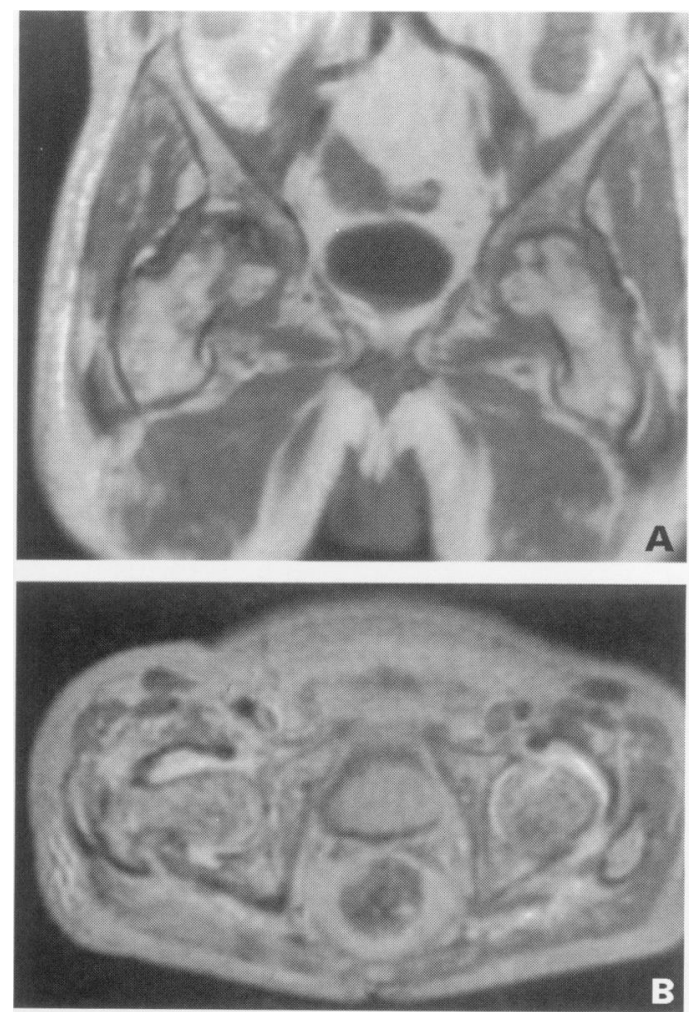

Figure 2 (A) Coronal T1-weighted MRI; (B) axial T2-weighted MRI

J P Y Lay

R W Whitehouse

Correspondence to Dr J P Y Lay, Consultant Radiologist,

Royal Preston Hospital,

Sharoe Green Lane,

Fulwood, Preston PR2 9HT,

UK

Accepted 23 April 1998

\section{Questions}

1 What are the significant observations on the X-rays and MRI scan ?

2 What is the diagnosis? 


\section{Answers}

QUESTION 1

The plain films showed a rapid and progressive symmetrical reduction in the superior joint spaces of both hips (figure 1). MRI (0.5T MR Max, GE Medical Systems, Slough, UK) of the hips showed reduced signal intensity in the femoral heads and acetabular roofs bilaterally (compared with the adjacent normal highsignal bone marrow) with intermediate signal joint effusions in the T1-weighted images. In addition, there is marked loss of the articular cartilage and irregularity, particularly of the right femoral head (figure 2A). T2-Weighted images showed patchy increased signal intensity in the femoral heads and high signal joint effusions bilaterally (figure $2 \mathrm{~B}$ ).

\section{QUESTION 2}

The appearances are highly suggestive of bilateral septic arthritis. Avascular necrosis, rheumatoid arthritis, sero-negative arthropathy and idiopathic chondrolysis of the hips should all be considered in the differential diagnosis, but none of these conditions are likely in the present clinical context. We aspirated $20 \mathrm{ml}$ of frank pus from both hip joints under ultrasound guidance, from which Escherichia coli was cultured. The infection was treated by bilateral open arthrotomy and washout and intravenous antibiotics.

\section{Discussion}

Septic arthritis is a recognised complication of immunosuppression on long-term steroid therapy. It is often a diagnostic problem for clinicians and radiologists, and debilitating complications such as total destruction of a joint, osteonecrosis and ankylosis may occur as a result. Polyarticular involvement occurs in $9 \%$ of patients with bacterial arthritis, ${ }^{1}$ but a bilateral, symmetrical pattern of infection is rare and can cause diagnostic confusion.

The first radiographic finding of septic arthritis is symmetrical soft tissue swelling around the involved joint. The hypertrophied synovium and presence of fluid cause widening of the joint space in joints with a tight capsule. The next radiographic finding is that of marginal erosions and loss of the joint space due to articular cartilage destruction. This is followed by the loss of the white cortical line

1 Kelly PJ. Bacterial arthritis in the adult. Orthop Clin North Am 1975;6:973-81.

2 Brower AC. Septic arthritis. Radiol Clin North Am 1996;34: 293-309.

3 Weissman BN, Hussain S. Magnetic resonance imaging of the knee. Rheum Dis Clin North Am 1991;17:637-68. over a contiguous segment, considered to be the hallmark of septic arthritis. ${ }^{2}$ Finally there is destruction of the underlying bone. Pyogenic septic joint usually causes rapid joint space loss with aggressive erosive changes and preservation of mineralisation, whereas the tuberculous septic joint tends to preserve the joint and causes marginal erosions and extensive demineralisation with little repair.

MRI, where available, is now the imaging investigation of choice in suspected septic arthritis. A joint effusion is seen as a region of low signal intensity on T1- and high signal intensity on T2- weighted images. However, MRI cannot distinguish septic fluid from inflammatory non-septic fluid. ${ }^{3}$ Cartilage destruction may be demonstrated on MRI, and marrow oedema at the articular ends of the bones within the septic joint is seen as high signal on T2- and low signal on T1-weighted images. Erosion of the bone appears as low signal intensity on T1-weighted images. Although MRI is highly sensitive and can demonstrate abnormalities within 24 hours of the onset of septic arthritis, it still lacks specificity and the final diagnosis is made only by culture and aspiration of the joint fluid.

Articular infections with $E$ coli and other Gram-negative bacilli are rare except in the intravenous drug abuser, those with preexisting joint disease and those with a chronic debilitating disorder. ${ }^{4}$ The radiographic changes of rapid joint space narrowing and cartilage destruction are highly unusual for avascular necrosis, rheumatoid arthritis, seronegative arthropathy, crystal arthropathy or pigmented villonodular synovitis. Idiopathic chondrolysis of the hip is rare, usually affects a much younger age group and is commonly mono-articular, although it is reported to be bilateral in $5 \%$ of cases. ${ }^{5}$ This case illustrates that a bilateral, symmetrical pattern of joint involvement does not exclude infection, and joint complications other than avascular necrosis may occur in patients on long-term steroid therapy.

\section{Final diagnosis}

Bilateral septic arthritis of the hips.

Keywords: septic arthritis; hip joint; MRI; Escherichia coli; steroid therapy

\footnotetext{
4 Lopitaux R, Sirot J, Melonx J, et al. Bone and joint infections due to gram-negative bacilli. Rev Rheum Mal Osteoartic 1982;49:187.

5 Jones BS. Adolescent chondrolysis of the hip joint. $S$ Afr Med f $1971 ; 45: 196-202 .^{2}$
} 